\title{
Uncorrelatedness in growing networks with preferential survival of nodes
}

\author{
David Juher* and Joan Saldaña ${ }^{\dagger}$ \\ Departament d'Informàtica i Matemàtica Aplicada, \\ Universitat de Girona, 17071-Girona, Spain
}

\begin{abstract}
The emergence of uncorrelated growing networks is proved when nodes are removed either uniformly or under the preferential survival rule recently observed in the WWW evolution. To this aim the rate equation for the joint probability of degrees is derived and stationary symmetrical solutions are obtained by passing to the continuum limit. When uniformly random removal of extant nodes and linear preferential attachment of new nodes are at work, we obtain that the only stationary solution corresponds to uncorrelated networks for any removal rate $r \in[0,1)$. In the more general case of preferential survival of nodes, uncorrelated solutions are also obtained. These results generalize the uncorrelatedness displayed by the (undirected) Barabási-Albert network model to models with uniformly random and selective (against low degrees) removal of nodes.
\end{abstract}

PACS numbers: 89.75.Fb, 87.23.-n, 87.23.-n, 89.75.Hc

*Electronic address: juher@ima.udg.edu

${ }^{\dagger}$ Electronic address: joan.saldana@udg.edu 


\section{INTRODUCTION}

Since the publication of the influential papers $[1,2]$, complex networks have attracted a lot of attention from scientists in different fields. From the pure beginning it has been a remarkable effort devoted to predict the implications of different mechanisms of addition and removal of nodes for the structure and functionality of the resulting network. Selective node deletion affecting mainly nodes with a high connectivity has been associated to intentional attacks, whereas uniformly random deletion is associated to random failures of nodes $[3,4]$. Moreover, a recent empirical study on time evolution of large data sets of the World Wide Web (WWW) concludes that, considering the WWW as an undirected network, the deletion probability of a given page (node) decreases with the number of links to other pages (node degree) according to a power law [5]. This suggests to the authors that a new mechanism, called preferential survival, is at work in the evolution of the WWW. The same mechanism has been also recently introduced in [6] for a food-web evolutionary model, and a somehow similar mechanism was proposed in [7] for the deactivation probability of nodes in citation networks.

The consequences of a uniformly random deletion have been widely analyzed for different models and some of its implications are well understood [8-13]. On the contrary, network models involving selective removal are far more complicated to analyze [14] and some caution must be taken before trusting some results extracted from models in which correlations have not been considered (see, for instance, [5]). The main difficulty for solving these models lies in the fact that the consequences of deletion on the degree evolution of nodes cannot be evaluated without a priori knowledge of some topological features of the network as, in particular, the degree correlations. A especially simple case that allows to overcome this difficulty is given by the assumption that correlations are absent (uncorrelated networks). Of course, one has to prove that such an assumption remains true as the network evolves which, in general, will not be the case. Indeed, a revealing recent work has shown that negative degree-degree correlations in highly heterogeneous real networks are to be expected in the absence of specific constraints [15]. In particular, for some empirical scale-free networks, the negative value of the Pearson's coefficient for the degree correlation is close to the value of the configuration of maximum total entropy of the expected values of the elements of the adjacency matrix for a given degree sequence $\left\{k_{i}\right\}_{i=1}^{N}$ under the assumption of a scale- 
free degree distribution. In this sense, other previous works $[16,17]$ showed that having at most one link per pair of nodes induces some negative correlation which in general is weaker than those observed in real networks [15]. Hence, for some scale-free networks, negative correlations can be explained without invoking anticorrelating mechanisms. In the framework of evolving networks under selective removal of nodes, the interplay between the degree evolution and degree correlations comes into play and offers a challenging problem when one aims to explain the origin of topological features as degree correlations. Certainly, stationary networks arising from mechanisms of addition and deletion of nodes will not fulfill any principle of maximum entropy. Hence, a first step is to see which mechanisms acting during the network growth are (or are not) able to render correlated networks.

\section{THE DISCRETE RATE EQUATION FOR $e_{i j}$}

Let us consider a general growing network which evolves, at each time $t>0$, under the addition and deletion of nodes. Precisely, a new node is connected to the network by means of $m$ new links. Each new link attaches to an extant node with a probability which depends only on its degree $k$. Following [14], this probability will be denoted by $\Pi_{k}^{a}(t)$. Moreover, with probability $0 \leq r<1$, a node is removed from the network (and all the edges incident to the removed node are also removed). A particular node will be removed with a probability $\Pi_{k}^{d}(t)$ which depends only on its degree $k$. If $n_{k}(t)$ is the number of nodes with degree $k$ at time $t$ in the network, then $\Pi_{k}^{a}(t) n_{k}(t)$ and $\Pi_{k}^{d}(t) n_{k}(t)$ are, respectively, the probability that the newly added node is connected to some node of degree $k$ and the probability of deleting some node of degree $k$ (provided a removal occurs, with probability $r$ ).

Let $p_{k}(t)$ be the degree distribution at time $t$. Consider the mean degree $\bar{k}(t)=\sum_{k} k p_{k}(t)$ and the expected degree of the removed node $\overline{k^{d}}(t)=\sum_{k} k \Pi_{k}^{d}(t) n_{k}(t)$ at time $t$. Denote by $N(t)$ and $L(t)$ the expected number of nodes and links in the network at time $t$, respectively. Without loss of generality we will assume that $N(0)=L(0)=0$, so that $N(t)=(1-r) t$. From now on we will assume that the degree distribution $p_{k}(t)$ stabilizes to a constant function for large values of $t$. It is well known that this assertion is true for a wide class of growing networks, including those obtained via preferential attachment mechanisms combined with either uniform deletion [13] or preferential survival of nodes (see [5] where the existence of such a convergence is claimed for $\alpha=1$ ). In this framework and since the stationary 
regime is attained very fast during the network growth (see Fig. 1), from now on we will drop the time dependence from the mean degree and the expected degree of the removed node and will simply write $\bar{k}$ and $\overline{k^{d}}$. In particular, from the evolution rules it follows that $L(t)=\left(m-r \overline{k^{d}}\right) t$ and, hence, the following useful relationship between $\bar{k}$ and $\overline{k^{d}}$ holds for $t$ large enough:

$$
2\left(m-r \overline{k^{d}}\right)=(1-r) \bar{k}
$$

To reinforce the validity of these assumptions we have run some Monte Carlo simulations. With this respect, it is worth to notice that the preferential survival mechanism is not well defined because the deletion probability is not well defined for singletons (nodes with degree 0 ), which in general can appear during the network growth, especially for low values of $m$. What is natural in order to complete the algorithmic definition of the preferential survival mechanism is to assign a deletion probability of 1 to every singleton. So, at each time step our algorithm searches for the existence of singletons and removes them. In Fig. 1 we have plotted the time evolution of $\bar{k}$ and $\overline{k^{d}}$ for networks with a final size of $10^{6}$ nodes, with $m=10$, a deletion rate $r=0.1$, and $\alpha=0.5,1$ and 2, as well as the corresponding theoretical prediction for the mean degree. We remark that $\overline{k^{d}}(t)$ has been computed as the mean of the degrees of all deleted nodes from the beginning of the simulation until time $t$. In all cases, the simulations provide numerical evidences of a rapid convergence of the degree distribution, reflected by a rapid convergence of both averages, and the validity of the approximation (1) even for small values of $t$, i.e., for network sizes much smaller than $10^{6}$.

Clearly, $\Pi_{k}^{a}$ and $\Pi_{k}^{d}$ depend on the particular addition and deletion mechanisms assumed in the model. However, next we start by deriving a completely general rate equation for the probability $e_{j k}$ that a randomly selected (directed) edge goes from a node of degree $j$ to a node of degree $k$. Since we are not assuming any inherent orientation of edges, the symmetry condition $e_{j k}=e_{k j}$ must be satisfied and each non-directed edge is counted twice. Moreover, by definition of joint probability distribution, $e_{j k}$ satisfies $\sum_{l} e_{l k}=k p_{k} / \bar{k}$, which is the probability of reaching a node of degree $k$ when a directed edge is randomly selected. Recall also that for uncorrelated networks the degree of the nodes at each end of any edge are statistically independent variables. Hence, for uncorrelated networks,

$$
e_{j k}=\frac{j p_{j}}{\bar{k}} \cdot \frac{k p_{k}}{\bar{k}}
$$


According to these ingredients and taking into account that $2 L(t) e_{j k}(t)$ is the expected number of directed edges of type $(j, k)$ at time $t$, it follows that

$$
2 L(t+1) e_{j k}(t+1)=2\left(L(t)+m-r \overline{k^{d}}\right) e_{j k}(t+1) .
$$

On the other hand, we can write the following discrete-time balance equation for the evolution of $e_{j k}$ neglecting higher-order degree correlations:

$$
\begin{aligned}
2(L(t) & \left.+m-r \overline{k^{d}}\right) e_{j k}(t+1)= \\
& =2 L(t) e_{j k}(t)+m \Pi_{k-1}^{a} n_{k-1} \delta_{j m}+m(k-1) e_{j \mid k-1} \Pi_{k-1}^{a} n_{k-1}-m k e_{j \mid k} \Pi_{k}^{a} n_{k} \\
& -r k e_{j \mid k} \Pi_{k}^{d} n_{k}-r \sum_{l}(k-1) e_{j \mid k} l e_{k \mid l} \Pi_{l}^{d} n_{l}+r \sum_{l} k e_{j \mid k+1} l e_{k+1 \mid l} \Pi_{l}^{d} n_{l}+\Delta_{2}
\end{aligned}
$$

where some dependencies on $t$ have been omitted for clarity and $e_{k \mid j}$ denotes the conditional probability that an edge that emanates from a node of degree $j$ reaches a node of degree $k$ :

$$
e_{k \mid j}=\frac{e_{j k}}{j p_{j} / \bar{k}}=\bar{k} N \frac{e_{j k}}{j n_{j}} .
$$

The second term in the rhs of (3) corresponds to a newly created $(m, k)$-edge arising from the attachment of the new node to a node of degree $k-1$. Note that there is not a loss term of the form $-m \Pi_{k}^{a} n_{k}$ associated to the attachment of the new node to a node of degree $k$ since this process cannot destroy edges that did not exist before the attachment (cf. [14]). The third and fourth terms in the rhs represent, respectively, the gain and loss of $(j, k)$-edges when attaching the new node to a node of degree $k-1$ which was previously linked to a node of degree either $j$ or $k$ that was at the end of a $(j, k)$-edge. The fifth term is the loss of $(j, k)$-edges due to the removal of the node with degree $k$ at one of its ends. Note also that a gain term of the form $r k e_{j+1 \mid k} \prod_{k}^{d} n_{k}$ cannot be present since the deletion of a node of degree $k$ at the end of a $(j+1, k)$-edge does not contribute to the creation of $(j, k)$-edges (cf. [14]). The last two terms in the rhs of (3) represent the loss and the gain of $(j, k)$-edges caused by deleting a neighbour of the node of degree $k$ at the end of a $(j, k)$-edge or a neighbour of a node of degree $k+1$ at the end of a $(j, k+1)$-edge, respectively. Finally, $\Delta_{2}$ contains the terms obtained by interchanging $j$ and $k$ in all the previous terms and, hence, contains the previous contributions affecting the node of degree $j$ at the other end of a $(j, k)$-edge.

When the degree distribution and the degree-degree correlation become stationary, $e_{j k}(t+$ 
1) $=e_{j k}(t)$, and using Eq. (4) the previous equation reads as:

$$
\begin{aligned}
2(m & \left.-r \overline{k^{d}}\right) e_{j k}=m \Pi_{k-1}^{a} n_{k-1} \delta_{j m}+m \bar{k} N\left(e_{j, k-1} \Pi_{k-1}^{a}-e_{j k} \Pi_{k}^{a}\right) \\
& -r \bar{k} N\left(e_{j k} \Pi_{k}^{d}+\bar{k}(k-1) \frac{e_{j k}}{k p_{k}} \sum_{l} e_{k l} \Pi_{l}^{d}-\bar{k} k \frac{e_{j, k+1}}{(k+1) p_{k+1}} \sum_{l} e_{k+1, l} \Pi_{l}^{d}\right)+\tilde{\Delta}_{2}
\end{aligned}
$$

where, similarly as before, $\tilde{\Delta}_{2}$ represents those terms obtained by interchanging $j$ and $k$ in the rhs of (5). For specific choices of attachment and deletion rules, the previous equation can be rewritten by taking the continuum limit for the degree.

\section{THE CONTINUOUS RATE EQUATION UNDER UNIFORM DELETION}

Under a uniformly random deletion of nodes, $\Pi_{k}^{d}=1 / N$ and, hence, $\overline{k^{d}}=\bar{k}$. Thus, from Eq. (5) we get

$$
\begin{aligned}
2(m & -r \bar{k}) e_{j k}=m\left(\Pi_{k-1}^{a} n_{k-1} \delta_{j m}+\Pi_{j-1}^{a} n_{j-1} \delta_{k m}\right) \\
& +m \bar{k} N\left(e_{j, k-1} \Pi_{k-1}^{a}-e_{j k} \Pi_{k}^{a}+e_{j-1, k} \Pi_{j-1}^{a}-e_{j k} \Pi_{j}^{a}\right) \\
& -r \bar{k}\left(j\left(e_{j k}-e_{j+1, k}\right)+k\left(e_{j k}-e_{j, k+1}\right)\right)
\end{aligned}
$$

which is a difference equation for $e_{j k}$ in both subscripts. Now, taking the approximation $\partial_{j} e(j, k) \approx e_{j+1, k}-e_{j k}$ and $\partial_{k} e(j, k) \approx e_{j, k+1}-e_{j k}$, we can pass to the continuum limit and write, for $j, k>m$, the previous equation as

$$
\begin{aligned}
2(m-r \bar{k}) e & =r \bar{k}\left(j \frac{\partial e}{\partial j}+k \frac{\partial e}{\partial k}\right) \\
& -m \bar{k} N\left(\frac{\partial\left(e \Pi_{j}^{a}\right)}{\partial j}+\frac{\partial\left(e \Pi_{k}^{a}\right)}{\partial k}\right)
\end{aligned}
$$

where $e(j, k)$ is a function of two variables such that $e(j, k)=e(k, j)$. Moreover, since it is a joint probability density function, it must satisfy $\int_{m}^{\infty} \int_{m}^{\infty} e(j, k) d j d k=1$.

Moreover, in the particular case of linear preferential attachment (LPA), $\Pi_{k}^{a}=k /(\bar{k} N)$ and Eq. (7) now simply reduces to

$$
j \frac{\partial e}{\partial j}+k \frac{\partial e}{\partial k}=-\frac{4}{1-r} e,
$$

where we have used that $\bar{k}=2 m /(1+r)$ [13]. This linear first-order PDE for $e(j, k)$ can be easily solved by separation of variables, and a unique solution of the form $e(j, k)=J(j) K(k)$ 
follows from imposing that $e(j, k)=e(k, j)$ and the normalizing condition. More precisely, if we introduce this expression for $e(j, k)$ into Eq. (7) and integrate the differential equations for $J(j)$ and $K(k)$, one gets that $e(j, k)=\left(C_{1} j^{\lambda-2 \beta}\right)\left(C_{2} k^{-\lambda}\right)$ where $\beta=2 /(1-r), C_{1}, C_{2}$ are arbitrary integration constants and $\lambda$ is an arbitrary constant of separation. Now, from $e(j, k)=e(k, j)$ it follows that $\lambda=\beta$. Normalizing the solution one obtains that

$$
e(j, k)=C_{0} j^{-2 /(1-r)} k^{-2 /(1-r)}
$$

with $C_{0}=\left(\frac{1+r}{1-r}\right)^{2} m^{\frac{2(1+r)}{1-r}}$. Finally, introducing the expression of $\bar{k}$ into $C_{0}$, the solution to (8) we have obtained can be written as

$$
e(j, k)=\frac{2 j}{\bar{k}(1-r) m}\left(\frac{j}{m}\right)^{-\frac{3-r}{1-r}} \frac{2 k}{\bar{k}(1-r) m}\left(\frac{k}{m}\right)^{-\frac{3-r}{1-r}} .
$$

On the other hand, for these type of growing networks, the resulting degree distribution was derived in [12] under the continuum approach for the rate equation of $n_{k}(t)$ and without any hypothesis about correlations. Precisely, it was obtained that

$$
p_{k}=\frac{2}{(1-r) m}\left(\frac{k}{m}\right)^{-\frac{3-r}{1-r}} .
$$

Remarkably, this degree distribution is in agreement with the marginal probability densities of $e(j, k)$ which are given by

$$
\int_{m}^{\infty} e(j, k) d j=\int_{m}^{\infty} e(k, j) d j=\frac{k p_{k}}{\bar{k}} \quad \forall k \geq m
$$

with $p_{k}$ given by (10). Note that, although there are other (positive and symmetrical) solutions to Eq. (8) that can be normalized to 1, only (9) has the previous marginal probability densities. Therefore, uncorrelated networks are the only configuration arising from the processes of PA and uniformly random deletion. With this respect, it is worth of noticing that both Eq. (8) and (10) were derived without any assumption on the network architecture.

This result is -as far as we know- the first one that proves the non-existence of degreedegree correlations in growing networks with uniformly random deletion of nodes by showing the statistical independence of the degrees at the ends of a randomly selected edge, i.e., without using the surrogate function given by the average degree of the nearest neighbors as a function of the node degree [13]. Such an existence of uncorrelated networks, moreover, has been also checked by simulations with different values of the deletion rate $r$. 


\section{THE CONTINUOUS RATE EQUATION FOR UNCORRELATED NET- WORKS}

The previous example illustrates an ideal situation in which a first-order linear PDE can be easily derived without using any assumption on the degree-degree correlations in the network. It follows that the uncorrelated solution is the only stationary state during the network's growth and it yields the degree distribution which is compatible with the uncorrelated network. In general, however, more complex probabilities $\Pi_{k}^{d}$ will come into play and an extra hypothesis has to be introduced beforehand in order to evaluate the sums in Eq. (5) with respect to $l$ and get a PDE for $e(j, k)$. The idea is to look for uncorrelated solutions, i.e. solutions that factorize in the form $e(j, k)=\left(j p_{j} / \bar{k}\right) \cdot\left(k p_{k} / \bar{k}\right)$, from which we can obtain the degree distributions $p_{k}$ (if any) compatible with the lack of degree-degree correlations.

Under the hypothesis of statistical independence of the degrees at the ends of a randomly selected edge, the difference equation (5) for the stationary distribution of edges becomes:

$$
\begin{aligned}
2(m & \left.-r \overline{k^{d}}\right) e_{j k}=m\left(\Pi_{j-1}^{a} n_{j-1} \delta_{k m}+\Pi_{k-1}^{a} n_{k-1} \delta_{j m}\right) \\
& +m \bar{k} N\left(e_{j, k-1} \Pi_{k-1}^{a}-e_{j k} \Pi_{k}^{a}+e_{j-1, k} \Pi_{j-1}^{a}-e_{j k} \Pi_{j}^{a}\right) \\
& -r \overline{k^{d}}\left(j\left(e_{j k}-e_{j+1, k}\right)+k\left(e_{j k}-e_{j, k+1}\right)-2 e_{j k}\right) \\
& -r \bar{k} N\left(e_{j k} \Pi_{j}^{d}+e_{j k} \Pi_{k}^{d}\right) .
\end{aligned}
$$

Approximating the differences by the corresponding partial derivatives we can pass to the continuum limit and write, for $j, k>m$, the PDE that must satisfy the stationary joint probability density function $e(j, k)$ of any uncorrelated growing network whose evolution is governed by attachment and deletion probabilities $\Pi_{k}^{a}$ and $\Pi_{k}^{d}$ :

$$
\begin{aligned}
2\left(m-r \overline{k^{d}}\right) e= & -m \bar{k} N\left(\frac{\partial\left(e \Pi_{j}^{a}\right)}{\partial j}+\frac{\partial\left(e \Pi_{k}^{a}\right)}{\partial k}\right) \\
& +r \overline{k^{d}}\left(j \frac{\partial e}{\partial j}+k \frac{\partial e}{\partial k}+2 e\right) \\
& -r \bar{k} N\left(\Pi_{j}^{d}+\Pi_{k}^{d}\right) e .
\end{aligned}
$$

Solving this linear first-order PDE by separation of constants (for those $\Pi_{k}^{a}$ and $\Pi_{k}^{d}$ that make it possible) and imposing the normalizing and symmetry conditions, we find a solution that, if feasible, gives a degree distribution compatible with the uncorrelatedness of the network. 
In particular, note that Eq. (7), which was obtained without any prior assumption on the correlations, immediately follows from Eq. (12) under uniform deletion, since in this case $\Pi_{k}^{d}=1 / N$ and $\overline{k^{d}}=\bar{k}$.

\section{A. Uncorrelatedness under preferential survival}

Now, let us consider that the node deletion rule is given by the so-called preferential survival (PS) mechanism [5]. In this case, the probability of deleting a node of degree $k$ is proportional to $k^{-\alpha}$ with $\alpha>0$ being a tuning parameter, leading to $\Pi_{k}^{d}=k^{-\alpha} /\left(\overline{k^{-\alpha}} N\right)$. A direct calculation shows that $\overline{k^{d}}=\overline{k^{1-\alpha}} / \overline{k^{-\alpha}}$. Hence, the total number of links can be approximated by the asymptotic value $L(t)=\left(m-r \overline{k^{1-\alpha}} / \overline{k^{-\alpha}}\right) t$. Assuming LPA, introducing $\Pi_{k}^{d}$ in Eq. (12) and using (1), we obtain

$$
j \frac{\partial e}{\partial j}+k \frac{\partial e}{\partial k}=-\left(4+\frac{2 r}{\overline{k^{-\alpha}}(1-r)}\left(j^{-\alpha}+k^{-\alpha}\right)\right) e .
$$

Because $\overline{k^{-\alpha}}$ is constant in the stationary regime and we are looking for uncorrelated solutions, this PDE is easily solvable by separation of variables, i.e., looking for a solution of the form $e(j, k)=J(j) K(k)$. Recalling that Eq. (2) holds for uncorrelated networks, the stationary degree distribution will be given by $p_{k}=\bar{k} K(k) / k$. Therefore, after solving the PDE and imposing the symmetry condition, it follows that the constant of separation is $\lambda=-2$ and that the degree distribution compatible with uncorrelatedness is

$$
p_{k}=C \exp \left(\frac{\xi k^{-\alpha}}{\alpha}\right) k^{-3}
$$

where $C$ is the normalizing constant and $\xi=\frac{2 r}{\overline{k^{-\alpha}}(1-r)}$. In particular, for $\alpha=1, C=$ $\xi^{2} /\left(\left(\frac{\xi}{m}-1\right) \exp (\xi / m)+1\right)$ and $p_{k}$ corresponds to the degree distribution obtained in [5] except for the value of $C$. Therefore, our analysis shows that the degree uncorrelation implicitly assumed in [5] to derive $p_{k}$ is preserved during the network growth under LPA and PS. Eq. (14) also says that, when $r>0$ and $\alpha \rightarrow 0$, the exponential term (which tends to infinity as $\alpha \rightarrow 0 \forall k>0$ ) modifies the shape of the degree distribution towards that of a power law with an exponent larger than 3 (cf. Eq. (10) for $\alpha=0$ ).

In order to test the lack of degree-degree correlations in networks generated via LPA plus PS, we have run some Monte Carlo simulations of the model for $m=10, \alpha=0.5,1,2$ and $r=0.1,0.3,0.5,0.7$. For each configuration of parameters $\{\alpha, r\}$ we have generated five 
networks of 150000 nodes, a size that is large enough for the network to be in the stationary regime. In all cases, the obtained value of the Pearson's coefficient for the degree correlation turns out to lie in the range $(0.0016,0.0123)$ and there is no trend in the values of this coefficient with $\alpha$ or $r$.

\section{DISCUSSION}

In this Article we have seen that preferential attachment of new nodes and preferential survival of existing ones give rise to uncorrelated networks for $\alpha \geq 0$. In the case of uniformly random deletion of nodes $(\alpha=0)$, this is the only type of networks predicted by the continuum approach and this fact has been supported by numerical simulations. In contrast, in the case of preferential survival $(\alpha>0)$, other configurations are, in principle, possible because the continuous rate equation (13) for the joint probability density function $e(j, k)$ was derived under the assumption of uncorrelation of degrees. These alternative network configurations, however, have been not observed in our simulations.

In addition to other rules for deleting nodes, mechanisms based on addition/deletion of edges can also induce degree correlations between neighboring nodes [5, 18-20]. In these cases, one should expect disagreement between the degree distributions computed assuming uncorrelation and those following from the solution to the rate equation for $e_{j k}$ by computing the marginal probability densities. This is what happens, for instance, with the existence of negative degree correlations in the Internet [15] that calls for new mechanisms at work during the network growth, in addition to those already considered in this paper. In this situation, uncorrelation is not preserved during the network growth and degree correlations must be taken into account in the derivation of degree distributions (cf. [5]).

Discrete rate equations for the joint probability $e_{j k}$ were initially introduced by $[21,22]$ to study the correlations between in-degrees and out-degrees in directed growing networks without deletion of nodes. The conclusion of these works, namely, the existence of correlations between both types of degrees in scale-free networks under preferential attachment, has been wrongly extrapolated to undirected growing networks by some authors $[18,19]$. In this Article, we have shown (and empirically validated) the existence of a family of models defined by preferential survival of extant nodes and linear preferential attachment of new ones which is able to yield uncorrelated networks. In this sense, a discrete rate equation 
has been recently used also for some authors to see under which mechanisms of addition and deletion of nodes undirected non-growing networks remain uncorrelated as long as they evolve [14]. Certainly, the assumption of having uncorrelated networks simplifies a lot the analytical treatment of the models but, on the other hand, it implies that one must check if such a property is preserved during the network development. Here we have presented a way to do this analysis which renders which degree distributions are compatible with uncorrelatedness under given rules of addition and deletion of nodes.

Acknowledgements. This work has been partially supported by the projects MTM200806349-C03-02 of the Spanish government (DJ and JS) and 2009SGR-345 of the Generalitat de Catalunya (JS).

[1] D. J. Watts and S. H. Strogatz, Nature 393, 440 (1998).

[2] A.-L. Barabási and R. Albert, Science 286, 509 (1999).

[3] R. Albert, H. Jeong, and A.-L. Barabási, Nature 406, 378 (2000).

[4] L. K. Gallos, R. Cohen, P. Argyrakis, A. Bunde, and S. Havlin, Physical Review Letters 94, 188701 (2005).

[5] J. S. Kong and V. P. Roychowdhury, Physica A 387, 3335 (2008).

[6] P. P. A. Staniczenko, O. T. Lewis, N. S. Jones, and F. Reed-Tsochas, Ecology Letters 13, 891 (2010).

[7] K. Klemm and V. M. Eguíluz, Phys. Rev. E 65, 036123 (2002).

[8] C. Cooper, A. Frieze, and J. Vera, Internet Mathematics 1, 463 (2004).

[9] F. Chung and L. Lu, Internet Mathematics 1, 409 (2004).

[10] C. Moore, G. Ghoshal, and M. E. J. Newman, Physical Review E 74, 036121 (2006).

[11] K. Deng, H. Zhao, and D. Li, Physica A 379, 714 (2007).

[12] J. Saldaña, Physical Review E 75, 027102 (2007).

[13] J. L. Garcia-Domingo, D. Juher, and J. Saldaña, Physica D 237, 640 (2008).

[14] B. Karrer and G. Ghoshal, Eur. Phys. J. B 62, 239 (2008).

[15] S. Johnson, J. J. Torres, J. Marro, and M. A. Muñoz, Phys. Rev. Lett. 104, 108702 (2010).

[16] J. Park and M. E. J. Newman, Phys. Rev. E 68, 026112 (2003).

[17] S. Maslov, K. Sneppen, and A. Zaliznyak, Physica A 333, 529 (2004). 
[18] S. N. Dorogotsev and J. F. F. Mendes, Evolution of networks: From biological nets to the Internet and $W W W$ (Oxford University Press, Oxford, UK, 2003).

[19] R. Xulvi-Brunet, W. Pietsch, and I. M. Sokolov, Phys. Rev. E 68, 036119 (2003).

[20] S. Johnson, J. Marro, and J. Torres, Journal of Statistical Mechanics: Theory and Experiment p. P03003 (2010).

[21] P. L. Krapivsky and S. Redner, Physical Review E 63, 066123 (2001).

[22] P. L. Krapivsky, G. J. Rodgers, and S. Redner, Phys. Rev. Lett. 86, 5401 (2001).

\section{Figure Caption}

Figure 1. Time evolution of the mean degree $(\bar{k}(t))$ and the average degree of deleted nodes $\left(\overline{k^{d}}(t)\right)$ in growing networks with LPA and PS for $\alpha=0.5$ (left), 1 (middle), and 2 (right). The final time is attained when the network size equals $10^{6}$. The values of the parameters are $m=10, r=0.1$. Solid lines correspond to the predicted value of $\bar{k}$ according to Eq. (14): $\bar{k}=18.67$ (left), 18.97 (middle), and 19.30 (right). Open circles correspond to the values of $\overline{k^{d}}(t)$ according to Eq. (1) using the observed values of $\bar{k}(t)$. 


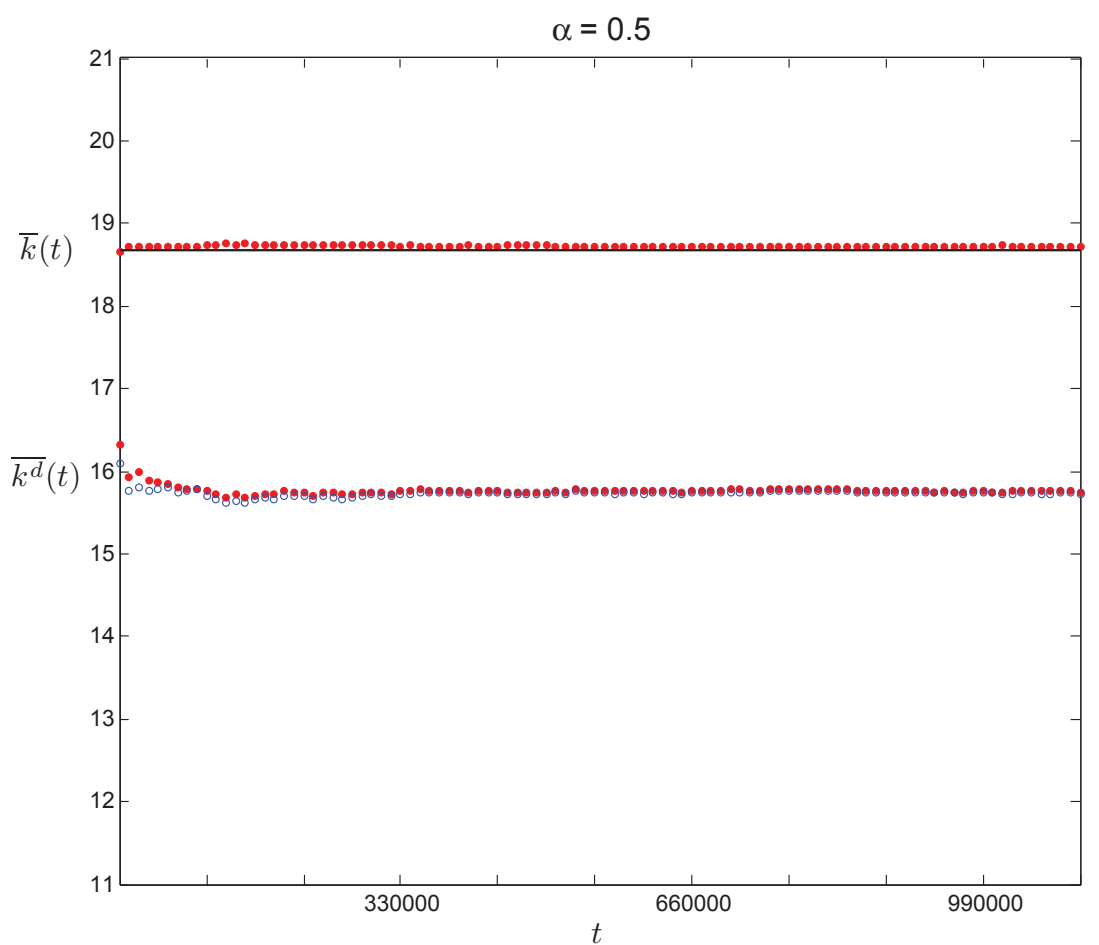

FIG. 1: Figure 1a (left) 


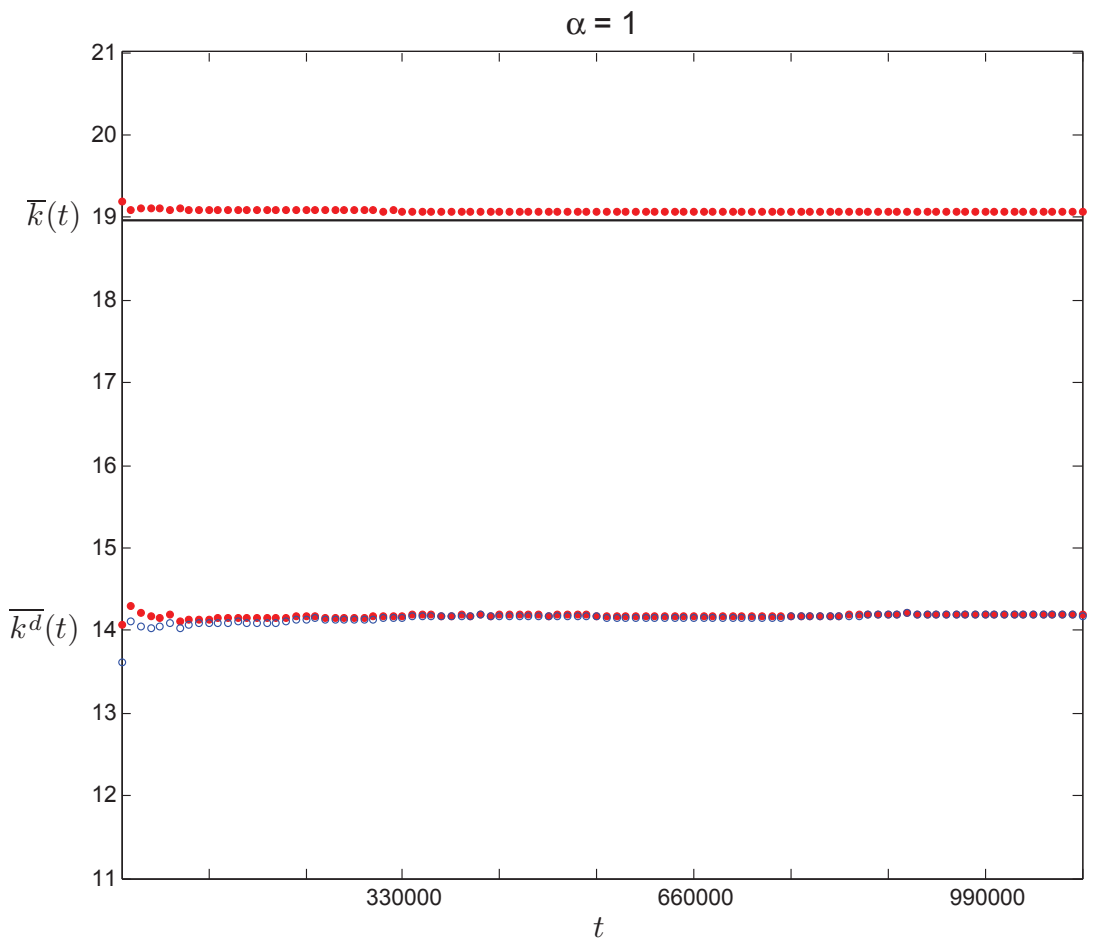

FIG. 2: Figure 1b (middle) 


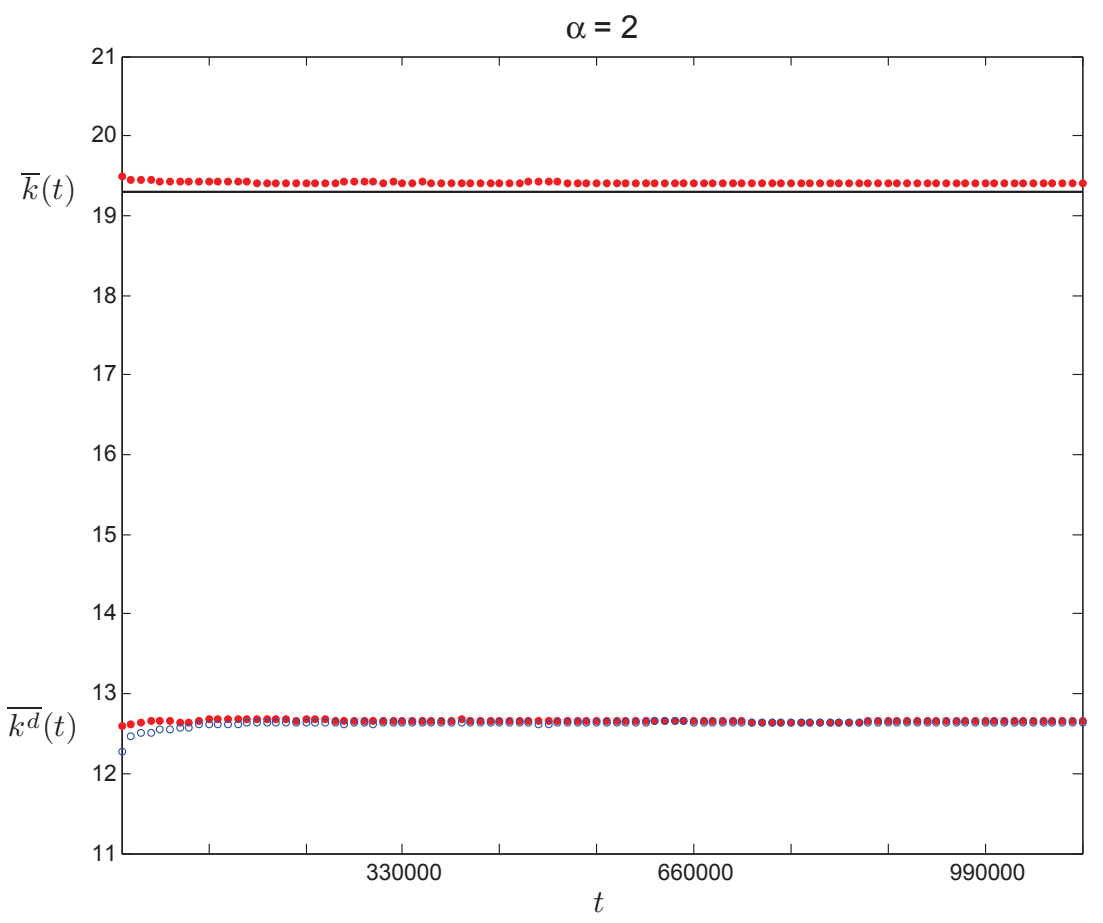

FIG. 3: Figure 1c (right) 